\title{
Direct-to-consumer genetic testing: a revised position statement of the American College of Medical Genetics and Genomics
}

\author{
ACMG Board of Directors ${ }^{1}$
}

Disclaimer: These recommendations are designed primarily as an educational resource for medical geneticists and other health-care providers to help them provide quality medical genetics services. Adherence to these recommendations does not necessarily assure a successful medical outcome. These recommendations should not be considered inclusive of all proper procedures and tests or exclusive of other procedures and tests that are reasonably directed to obtaining the same results. In determining the propriety of any specific procedure or test, geneticists and other clinicians should apply their own professional judgment to the specific clinical circumstances presented by the individual patient or specimen. It may be prudent, however, to document in the patient's record the rationale for any significant deviation from the recommendations.

Genet Med advance online publication 17 December 2015

Key Words: consumer; direct-to-consumer; genetic testing; self-testing; OTC
With ongoing genetic discoveries and improvements in technology, more genetic tests are available than ever before. Along with greater availability has come increased consumer demand for genetic tests and expansion of direct-to-consumer testing. The American College of Medical Genetics and Genomics (ACMG) has revised its 2008 e-publication regarding this issue (ACMG Statement on Direct-to-Consumer Genetic Testing, retired; available by request to acmg@acmg.net) and believes that it is critical for the public to realize that genetic testing is only one part of a complex process that includes genetic risk assessment, diagnosis, and disease management. Thus, the results of such testing can have important health implications for individuals and their family members. ACMG believes that the following should be considered the minimum requirements for genetic tests that can reveal health-related information:

- Genetic testing and the interpretation of results are highly technical and complex. The clinical testing laboratory must beaccreditedbytheCLIA(ClinicalLaboratoryImprovement Amendments) Program, the state, and/or other applicable accrediting agencies. The accreditation process ensures that laboratories adhere to strict standards and guidelines for clinical testing. Test result reports to consumers should indicate the specifics of the laboratory's accreditation. A knowledgeable professional should be involved in the process of ordering a genetic test with medical implications and laboratory results should be interpreted and delivered by a board-certified genetics professional.
- A genetics expert such as a certified medical geneticist or genetic counselor should be available to help the consumer determine, for example, whether a genetic test should be performed and how to interpret test results in light of personal and family history. A board-certified genetic counselor can help facilitate this process by providing information about the test and helping to explain test results. A number of risks can be reduced if a boardcertified genetics professional is involved in genetic testing, including inadequate or lack of informed consent, testing without the appropriate indications for testing, selecting inadequate methods for testing or selecting the wrong test, and misinterpretation of results leading to inappropriate choices for disease management or prevention and inadequate follow-up.

- The consumer should be fully informed regarding what the test can and cannot say about his or her health. Many direct-to-consumer genetic tests do not give a definitive answer regarding whether an individual will develop a given condition but instead only provide information about the risk or probability of developing a disease. The medical interpretation of such results is often complex and includes additional patient-specific information, such as prior medical and family history and other factors. This information needs to be incorporated and communicated to the consumer in the appropriate context and in an understandable fashion that is linguistically and culturally appropriate.

\footnotetext{
American College of Medical Genetics and Genomics, Bethesda, Maryland, USA. Correspondence: Michael S. Watson (mwatson@acmg.net)

Approved by the Board of Directors of the American College of Medical Genetics and Genomics on 24 August 2015.
}

Submitted 6 November 2015; accepted 6 November 2015; advance online publication 17 December 2015. doi:10.1038/gim.2015.190 
- The consumer should be apprised of the potential for receiving results that can neither confirm nor rule out the possibility of disease or unexpected results that are unrelated to the specific reason for testing, as well as the implications of genetic test results for family members.

- The scientific evidence base describing the validity and utility of a genetic test should be clearly stated. Direct-to-consumer genetic test providers should provide easy-to-understand information with primary references documenting the scientific data on which a specific test is based.

- Privacy concerns must be addressed. Prior to testing, the consumer should be informed as to who will have access to test results, what processes are in place to protect these results, what will happen to the DNA sample once testing is complete, and whether the test results may have any personal or family-related implications for life, long-term care, or disability insurance. Finally, whether data generated from testing will be sold to or shared with third parties should be clearly disclosed, as should ownership of the sample and generated data.

\section{DISCLOSURE}

The authors declare no conflict of interest. 